\title{
Effects of Carrier Phase Error on EGC Receivers in Correlated Nakagami- $m$ Fading
}

\author{
Nikos C. Sagias, Member, IEEE and George K. Karagiannidis, Senior Member, IEEE
}

\begin{abstract}
The effects of incoherently combining on dualbranch equal-gain combining (EGC) receivers in the presence of correlated, but not necessarily identical, Nakagami- $m$ fading and additive white Gaussian noise are studied. Novel closedform expressions for the moments of the output signal-to-noise ratio (SNR) are derived. Based on these expressions, the average output SNR and the amount of fading are obtained in closedform. Moreover, the outage and the average bit error probability for binary and quadrature phase-shift keying are also studied using the moments-based approach. Numerical and computer simulation results clearly depict the effect of the carrier phase error, correlation coefficient, and fading severity on the EGC performance. An interesting finding is that higher values of the correlation coefficient results to lower irreducible error floors.
\end{abstract}

Index Terms - Bit error rate (BER), carrier phase error, equalgain combining (EGC), Nakagami- $m$ fading, outage probability, phase-locked loop (PLL).

\section{INTRODUCTION}

D IGITAL communication receivers employing equal-gain combining (EGC) present significant practical interest, because they provide performance comparable to maximalratio combining receivers, but with simpler implementation complexity. In EGC, the received signals are co-phased, equally weighted, and then summed to form the resultant signal [1]. This letter focuses on EGC receivers operating in correlative fading. Among recently published papers on this topic, including [2]-[4], the usual assumption for perfect carrier phase estimation has been made. However, several impairments, such as Doppler spread in the carrier frequency and the presence of noise and interference in the carrier recovery loops do not allow for perfect estimation of the carrier phase. Hence, when carrier phase estimation errors are further assumed, only one paper has been published [5] in which the average bit error probability (ABEP) is approximated using the Gram-Charlier series expansion. However, in that useful work, identical and statistically independent Rayleigh fading has been considered.

In this paper, we extend [5] for the case of dual-branch EGC receivers operating over correlated, but not necessarily identically distributed, Nakagami- $m$ fading channels [1]. By deriving exact closed-form expressions for the moments of the output signal-to-noise ratio (SNR), we study: $i$ ) the average

Manuscript received October 12, 2004. The associate editor coordinating the review of this letter and approving it for publication was Prof. Ernesto Conte.

N. C. Sagias is with the Department of Physics, University of Athens, Panepistimiopolis, GR-15784 Athens, Greece (e-mail: nsagias@ space.noa.gr).

G. K. Karagiannidis is with the Electrical and Computer Engineering Department, Aristotle University of Thessaloniki, GR-54124 Thessaloniki, Greece (e-mail: geokarag@auth.gr).

Digital Object Identifier 10.1109/LCOMM.2005.07006 output SNR and the amount of fading (AoF) which are expressed in closed-forms, $i i)$ the outage probability and the ABEP for binary and quadrature phase-shift keying (BPSK and QPSK) modulation signallings which are investigated using the well-known moments-generating function (mgf) approach and the Padé approximants [4]. Numerical and computer simulations results demonstrate the impact of the correlation coefficient on the EGC performance, especially in cases where the effects of carrier phase error are not dominant.

\section{System And Channel Model}

Let $s$ be a transmitted signal of energy $E_{s}=\mathcal{E}\left\langle|s|^{2}\right\rangle$, with $s= \pm 1(M=2)$ for BPSK and $s= \pm \exp ( \pm \jmath \pi / 4)(M=4)$ for QPSK and with $M$ being the complex alphabet size $(\mathcal{E}\langle\cdot\rangle$ denotes statistical averaging and $\jmath=\sqrt{-1}$ is the complex operator), which is corrupted by correlated multiplicative fading and additive white Gaussian noise (AWGN). After multiplying the received signals of the respective channel by the complex conjugate of the phase-shift estimates, the complex baseband signal at the output of the dual-branch EGC receiver can be written as

$$
z=\sum_{i=1}^{2}\left\{s r_{i} \exp \left[\jmath\left(\varphi_{i}-\widehat{\varphi}_{i}\right)\right]+n_{i}\right\}
$$

where $n_{\ell}$ is the $\ell$ th AWGN envelope $(\ell=1$ and 2$)$ with a single-sided power spectral density $N_{0}$ identical to both diversity input channels. Moreover, $r_{1}$ and $r_{2}$ represent the correlated fading envelopes modelled as Nakagami- $m$ distributed random variables $(\mathrm{RV}) \mathrm{s}, \varphi_{\ell}$ is the random phaseshift introduced by the $\ell$ th channel assumed to be uniformly distributed in the interval $(-\pi, \pi]$, and $\widehat{\varphi}_{\ell}$ is the estimated phase of $\varphi_{\ell}$ at the receiver. The usual assumptions are made, that the channel fading is sufficiently slow to allow the implementation of coherent detection, the phase processes are slowly varying so that they are considered constant over one signaling interval, and that the carrier phase errors, caused by a first order phase-locked loop (PLL), follows the Tikhonov distribution.

Since $r_{\ell}$ is a Nakagami- $m$ RV, the instantaneous SNR per symbol at the $\ell$ th input branch $\gamma_{\ell}=r_{\ell}^{2} E_{s} / N_{0}$ is a Gamma distributed RV with a marginal probability density function (pdf) given by [1, eq. (2.21)]

$$
f_{\gamma_{\ell}}(\gamma)=\left(\frac{m}{\bar{\gamma}_{\ell}}\right)^{m} \frac{\gamma^{m-1}}{\Gamma(m)} \exp \left(-\frac{m}{\bar{\gamma}_{\ell}} \gamma\right) \text {. }
$$

In the above equation, $\bar{\gamma}_{\ell}$ and $m$ are the average SNR per symbol $\bar{\gamma}_{\ell}=\mathcal{E}\left\langle r_{\ell}^{2}\right\rangle E_{s} / N_{0}$ and the Nakagami- $m$ fading parameter, respectively, and $\Gamma(\cdot)$ is the Gamma function $[6$, 
eq. $(8.310 / 1)]$. The $(n+k)$ th order product moments of $\gamma_{1}$ and $\gamma_{2}$ are [4]

$\mathcal{E}\left\langle\gamma_{1}^{n} \gamma_{2}^{k}\right\rangle=\bar{\gamma}_{1}^{n} \bar{\gamma}_{2}^{k} \frac{\Gamma(m+n) \Gamma(m+k)}{m^{n+k} \Gamma^{2}(m)}{ }_{2} F_{1}(-n,-k ; m ; \rho)$

where $\rho$ is the correlation coefficient between envelopes $r_{1}$ and $r_{2}$ and ${ }_{2} F_{1}(\cdot, \cdot ; \cdot ; \cdot)$ is the Gauss hypergeometric function [6, eq. (9.100)].

Let $\psi_{\ell}$ denotes the phase estimation error, i.e., $\psi_{\ell}=\varphi_{\ell}-$ $\widehat{\varphi}_{\ell}$. When Gaussian noise is present in the recovery loop, the steady-state pdf of the phase error in the $\ell$ th PLL, is given by

$$
f_{\psi_{\ell}}(\psi)=\frac{\exp \left[\zeta_{\ell} \cos (\psi)\right]}{2 \pi I_{0}\left(\zeta_{\ell}\right)}, \quad|\psi| \leq \pi
$$

where $I_{n}(\cdot)$ is the $n$th order modified Bessel of the first kind [6, Section 8.40] and $\zeta_{\ell}$ is the SNR in the $\ell$ th loop. When $\zeta_{\ell} \geq 10 \mathrm{~dB}$, the root mean square (RMS) phase error $\sigma_{\psi_{\ell}}=$ $\mathcal{E}\left\langle\overline{\psi_{\ell}^{2}}\right\rangle$ can be approximated as $\sigma_{\psi_{\ell}} \simeq 1 / \sqrt{\zeta_{\ell}}$.

\section{Statistics of ThE EGC OUtPUt SNR}

The instantaneous SNR per symbol at the output of the dual-branch EGC receiver can be written as

$$
\gamma_{e g c}=\frac{A^{2}}{2}\left[\sum_{i=1}^{2} \sqrt{\gamma_{i}} \cos \left(\psi_{i}+\xi\right)\right]^{2}
$$

where for BPSK, $A=1$ and $\xi=0$, while for QPSK, $A=\sqrt{2}$ and $\xi=-\pi / 4$.

\section{A. Moments of the Output SNR}

By definition and using (5), the $n$th order moment of the EGC output SNR per symbol, $\mu_{n}=\mathcal{E}\left\langle\gamma_{\text {egc }}^{n}\right\rangle$, can be expressed as

$$
\mu_{n}=\frac{A^{2 n}}{2^{n}} \mathcal{E}\left\langle\left[\sum_{i=1}^{2} \sqrt{\gamma_{i}} \cos \left(\psi_{i}+\xi\right)\right]^{2 n}\right\rangle
$$

By using the well-known binomial identity [6, eq. (1.111)], (6) can be written as

$$
\mu_{n}=(2 n) ! \frac{A^{2 n}}{2^{n}} \sum_{\substack{k_{1}=0 \\ k_{2}=2 n-k_{1}}}^{2 n} \mathcal{E}\left\langle\prod_{i=1}^{2} \frac{\gamma_{i}^{k_{i} / 2}}{k_{i} !} \cos ^{k_{i}}\left(\psi_{i}+\xi\right)\right\rangle
$$

In the above equation, the pairs $\gamma_{\ell}$ and $\psi_{\ell}$, as well as $\psi_{1}$ and $\psi_{2}$ are statistically independent, and thus, the above mean product can be rewritten as the product of three mean terms. The first two of them are the $k_{\ell}$ th order moment of $\cos \left(\psi_{\ell}+\xi\right)$ given by [5]

$\mathcal{E}\left\langle\cos ^{n}\left(\psi_{\ell}+\xi\right)\right\rangle=\frac{1}{2^{n}} \sum_{k=0}^{n}\left(\begin{array}{l}n \\ k\end{array}\right) \frac{I_{|2 k-n|}\left(\zeta_{\ell}\right)}{I_{0}\left(\zeta_{\ell}\right)} \cos [(2 k-n) \xi]$

while the third one is given by (3). Hence, by replacing (3) and (8) in (7), the $n$th moment of the EGC output SNR per symbol can be expressed in closed-form as

$$
\begin{aligned}
\mu_{n}= & \frac{A^{2 n}(2 n) !}{(8 m)^{n} \Gamma^{2}(m)} \sum_{\substack{k_{1}=0 \\
k_{2}=2 n-k_{1}}}^{2 n} F_{1}\left(-\frac{k_{1}}{2},-\frac{k_{2}}{2} ; m ; \rho\right) \\
& \times \prod_{i=1}^{2} \bar{\gamma}_{i}^{k_{i} / 2} \Gamma\left(m+\frac{k_{i}}{2}\right) \\
& \times \sum_{q=0}^{k_{i}}\left(\begin{array}{c}
k_{i} \\
q
\end{array}\right) \frac{I_{\left|2 q-k_{i}\right|}\left(\zeta_{i}\right)}{I_{0}\left(\zeta_{i}\right)} \cos \left[\left(2 q-k_{i}\right) \xi\right] .
\end{aligned}
$$

Note, that for $\bar{\gamma}_{1}=\bar{\gamma}_{2}, \rho=0$, and $m=1$, (9) reduces to an already known expression [5, eq. (61)] for independent and identically distributed Rayleigh fading channels.

1) Average output SNR: The EGC average output SNR per symbol $\bar{\gamma}_{e g c}$ is a useful performance measure serving as an excellent indicator of the overall system's fidelity. This measure can be obtained in closed-form by setting $n=1$ in (9) as $\bar{\gamma}_{e g c}=\mu_{1}$.

2) Amount of fading: The AoF, $A_{F} \triangleq \operatorname{var}\left(\gamma_{e g c}\right) / \bar{\gamma}_{e g c}^{2}$, is considered as a unified measure of the fading severity and is typically independent of the average fading power [1]. Using (9), the AoF at the EGC output can be easily expressed in a simple closed-form expression as $A_{F}=\mu_{2} / \mu_{1}^{2}-1$.

It is important to underline, that the higher order moments $(n \geq 3)$ are useful in signal processing algorithms for signal detection, classification and estimation since they play a fundamental role in analyzing the performance of wideband communications systems in presence of fading [7].

\section{B. MGF of the Output SNR}

The mgf of the EGC output SNR per symbol $\mathcal{M}_{\gamma_{e g c}}(s) \triangleq$ $\mathcal{E}\left\langle\exp \left(s \gamma_{e g c}\right)\right\rangle$ can be represented as a formal Taylor power series as $\mathcal{M}_{\gamma_{e g c}}(s)=\sum_{n=0}^{\infty} \mu_{n} s^{n} / n$ !. Although that the moments of all orders can be evaluated in closed-form (see (9)), we can not definitely conclude the number of terms which are needed in order to converge or whether it converges. The Padé approximants method is an efficient way for evaluating $\mathcal{M}_{\gamma_{\text {egc }}}(s)$ where practically only a few number of moments are used. A Padé approximant, is that rational function of polynomials of a specified order $N+1$ for the denominator and $N$ for the nominator which can accurately approximate $\mathcal{M}_{\gamma_{\text {egc }}}(s)$. For such a rational function, the first $(2 N+1)$ order moments are need in order to construct the approximated $M_{\gamma_{e g c}}(s)$. Note, that the Padé approximants method is available in most of the well-known mathematical software packages, such as Mathematica and Maple.

1) Outage probability: Let $\gamma_{t h}$ be a certain specified threshold. The outage probability is defined as the probability that $\gamma_{e g c}$ falls below $\gamma_{t h}$ and can be derived as

$$
P_{\text {out }}\left(\gamma_{t h}\right)=\left.\mathcal{L}^{-1}\left[\frac{\mathcal{M}_{\gamma_{e g c}}(-s)}{s} ; \gamma_{e g c}\right]\right|_{\gamma_{e g c}=\gamma_{t h}}
$$

where $\mathcal{L}^{-1}(\cdot ; \cdot)$ denotes the inverse Laplace transform. Due to the Padé's rational form of $\mathcal{M}_{\gamma_{e g c}}(s)$, the outage probability can be easily evaluated as

$$
P_{\text {out }}\left(\gamma_{t h}\right)=\sum_{i=1}^{N+1} \frac{\lambda_{i}}{p_{i}} \exp \left(p_{i} \gamma_{t h}\right)
$$


TABLE I

IRREDUCIBLE ERROR FLOORS FOR RMS PHASE ERRor $\sigma_{\psi}=20^{\circ}$. (BPSK AND $\bar{\gamma}_{1}=\bar{\gamma}_{2}$ )

\begin{tabular}{|c||c|c|c|}
\hline & $m=1$ & $m=2$ & $m=3$ \\
\hline \hline$\rho=0$ & $2.3810^{-6}$ & $7.1510^{-7}$ & $5.9610^{-7}$ \\
\hline$\rho=0.5$ & $1.7910^{-6}$ & $4.7610^{-7}$ & $2.7610^{-7}$ \\
\hline$\rho=0.9$ & $4.7610^{-7}$ & $1.510^{-7}$ & $10^{-7}$ \\
\hline \hline
\end{tabular}

where $p_{i}$ and $\lambda_{i}$ are the poles and the residues, respectively, of the Padé rational form of $M_{\gamma_{e g c}}(s)$ [4].

2) Average bit error probability: Using the Padé's rational form of $\mathcal{M}_{\gamma_{e g c}}(s)$ and in order to calculate the ABEP of BPSK and QPSK, simple integrals of the form

$$
\bar{P}_{b e}=\frac{g_{p s k}}{\pi} \int_{0}^{\pi-\pi / M} \mathcal{M}_{\gamma_{e g c}}\left[-\frac{\sin ^{2}(\pi / M)}{g_{p s k} \sin ^{2}(x)}\right] d x
$$

have to be numerically evaluated, where $g_{p s k}=1 / \log _{2}(M)$.

\section{Numerical AND Simulation Results}

The ABEP performance of the EGC is depicted in Figs. 1 and 2 for BPSK, $N=11$, and $\sigma_{\psi_{1}}=\sigma_{\psi_{2}}=\sigma_{\psi}$. In Fig. 1, $\bar{P}_{b e}$ is plotted as a function of $\bar{\gamma}=\bar{\gamma}_{1}=\bar{\gamma}_{2}, \sigma_{\psi}=20^{\circ}$, and several values for $m$ and $\rho$. As expected, an increase on the fading severity and / or $\rho$ leads to a decrease of $\bar{P}_{b e}$. For an increase of $\rho$ from 0 to 0.5 , an SNR penalty of about $2 \mathrm{~dB}$ is observed for $m=1$. Furthermore, as $m$ increases, the error floor due to phase error dominance appears at lower values of $\bar{P}_{b e}$ and surprisingly, for fixed values of $m$ and $\bar{\gamma}$, the higher value of $\rho$ the lower irreducible error floor appears. It should be mentioned, that for the special case of $\rho=0$ and $m=1$ the same results as that in [5, Fig. 3 and Table V] are obtained. In Fig. 2, $\bar{P}_{b e}$ is plotted as a function of $\sigma_{\psi}$ for a desired $\bar{\gamma}_{1}=15 \mathrm{~dB}, \bar{\gamma}_{2}=0.5 \bar{\gamma}_{1}$, and several values for $m$ and $\rho$. For medium to high values of $\sigma_{\psi}$ (e.g. $\sigma_{\psi}>$ $25^{\circ}$ ), the impact of phase error is significant, and hence, $\rho$ has minor effect on the ABEP. In contrary, when $\sigma_{\psi}<25^{\circ}$, the impact of the correlated fading channel dominates. For the special case of $\sigma_{\psi}=0^{\circ}$, same results with that in [4] are obtained. Monte Carlo simulations have been also performed and the corresponding results are included in Figs. 1 and 2 for comparison purposes. It is easily recognized, that there is a good match between numerical and computer simulations results. Finally, the decreasing irreducible error floor values with increasing $\rho$ are summarized in Table I.

\section{REFERENCES}

[1] M. K. Simon and M.-S. Alouini, Digital Communication over Fading Channels, 1st ed. New York: Wiley, 2001.

[2] M. Z. Win and R. K. Mallik, "Error analysis of noncoherent $M$-ary FSK with postdetection EGC over correlated Nakagami and Rician channels," IEEE Trans. Commun., vol. 50, pp. 378-383, Mar. 2002.

[3] R. K. Mallik, M. Z. Win, and J. H. Winters, "Performance of dualdiversity EGC in correlated Rayleigh fading with unequal branch SNRs," IEEE Trans. Commun., vol. 50, pp. 1041-1044, July 2002.

[4] G. K. Karagiannidis, "Moments-based approach to the performance analysis of equal gain diversity in Nakagami- $m$ fading," IEEE Trans. Commun., vol. 52, pp. 685-690, May 2004.

[5] M. A. Najib and V. K. Prabhu, "Analysis of equal-gain diversity with partially coherent fading signals," IEEE Trans. Veh. Technol., vol. 49, pp. 783-791, May 2000.

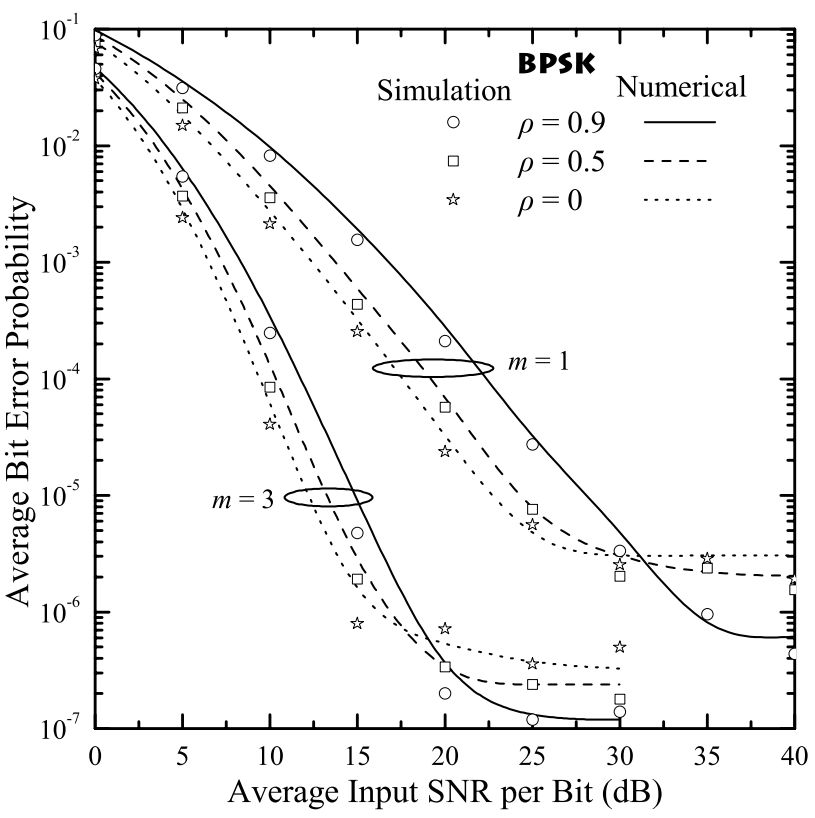

Fig. 1. ABEP of dual-branch EGC as a function of the average input SNR per bit for BPSK, equal average input channel and loop SNRs, and $\sigma_{\psi}=20^{\circ}$.

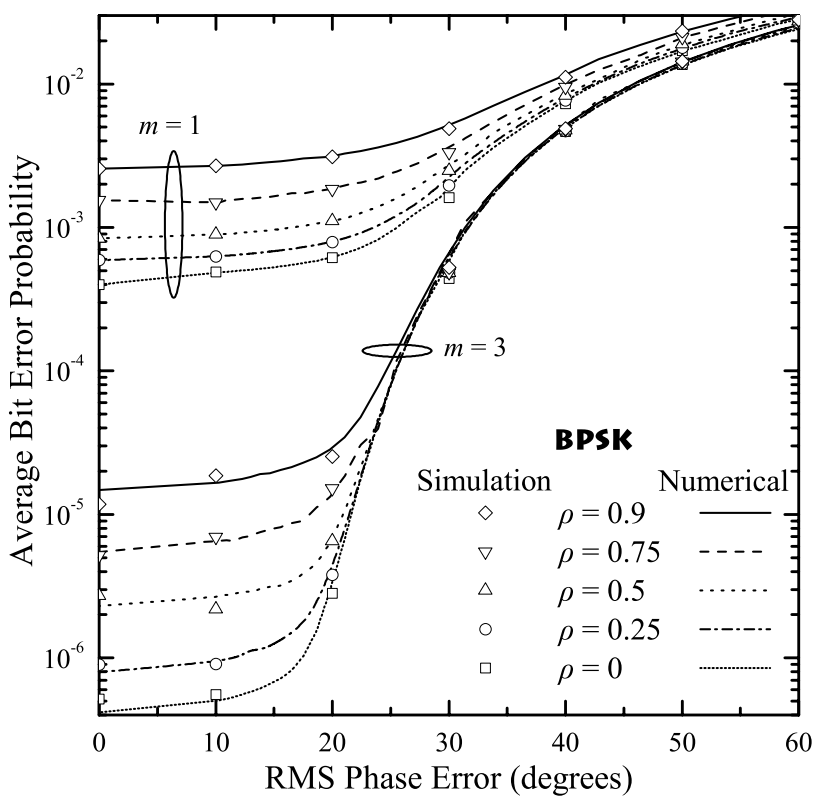

Fig. 2. ABEP of dual-branch EGC as a function of the RMS phase error for BPSK, unequal average input SNRs, desired $\bar{\gamma}_{1}=15 \mathrm{~dB}$, and $\bar{\gamma}_{2}=0.5 \bar{\gamma}_{1}$.

[6] I. S. Gradshteyn and I. M. Ryzhik, Table of Integrals, Series, and Products, 6th ed. New York: Academic, 2000.

[7] M. Z. Win, R. K. Mallik, and G. Chrisikos, "Higher order statistics of antenna subset diversity," IEEE Trans. Commun., vol. 2, pp. 871-875, Sept. 2003. 